\title{
Initiation of Basidioma Formation of Rare and Medicinal Macromycetes in Pure Culture
}

\author{
Maryna Sukhomlyn ${ }^{1}$, Vitaliia Didenko ${ }^{1}$, Nadiia Tsvyd $^{1 *}$, Yurii Petrychuk ${ }^{2}$ \\ ${ }^{1}$ Educational and Scientific Centre «Institute of Biology and Medicine», Taras Shevchenko National \\ University of Kyiv, Kyiv, Ukraine \\ ${ }^{2}$ Hutsulshchyna National Nature Park, Kosiv, Ukraine \\ Correspondence: suhmary@ukr.net
}

\begin{abstract}
More than 5000 species of Ascomycetes and Agaricales fungi are known nowadays. However, 30 species of them are intensively cultivated and have been used into the agriculture. One of the reasons of a small number of species that can be grown in industrial culture are unclear conditions for the basidiomes formation. Basidiomes and mycelium include various nutritional substances that are used in food, medicine, cosmetics, etc. Recently, the biosynthesis of fungal biologically active substances with immunomodulation, radioprotective, antitumor, antiviral and other properties is under investigation by many scientists. Only $15 \%$ of all products of medical mushrooms are made from extracts of mycelium. Verification of conditions of mushroom cultivation will significantly expand the range of species that could serve as a potential object for biotechnology.

29 species of macromycetes from the pure Cultures Collection of Fungi (FCKU) of Educational and Scientific Centre «Institute of Biology and Medicine» Taras Shevchenko National University of Kyiv were used in our experiment. The initiation basidiomes formation of 29 macromycetes on different substrates in pure culture was studied. The optimal substrate for the studied species was husk of sunflower seeds. Most fungi formed basidiomes on it. If mushrooms did not formed basidiomes on this substrate, they fastest it overgrown or developed primordia or sclerotium-shaped structures. The shaping of the basidiomes, primordia or sclerotiashaped structures was observed in 28 species. Three of these species were listed in the Red Book of Ukraine (Grifola frondosa, Leucoagaricus barsii, Sparassis crispa) and others rare species for Ukraine (Ceriporia viridans, Hericium cirrhatum, Sarcodontia crocea, Sparassis laminosa).
\end{abstract}

Key words: basidiomes formation, medicinal macromycetes, pure cultures, rare species, sclerotium-shaped structures.

\section{Ініціація формування плодових тіл рідкісних та лікарських грибів в умовах чистої культури}

\author{
Марина Сухомлин ${ }^{1}$, Віталія Діденко ${ }^{1}$, Надія Цвид ${ }^{1}$, Юрій Петричук² \\ ${ }^{1}$ Навчально-науковий центр «Інститут біології та медицини» Київського національного університету \\ імені Тараса Шевченка, Київ, Україна \\ ${ }^{2}$ Національний природний парк «Гуцульщина», Косів, Україна \\ Адреса для листування: suhmary@ukr.net
}

Отримано: 18.02.19; прийнято до друку:20.03.19; опубліковано: 28.06.19

Резюме. Вивчено ініціацію утворення плодових тіл 29 видів грибів на різних субстратах в умовах культури. У результаті скринінгу цих макроміцетів у 28 видів спостерігали формування плодових тіл, примордіїв або склероцієподібних структур. Також плодові тіла формували (Grifola frondosa, Leucoagaricus barsii, Sparassis crispa) три види, що занесені до Червоної книги України, та декілька рідкісних видів для України (Ceriporia viridans, Hericium cirrhatum, Sarcodontia crocea, Sparassis laminose).

Ключові слова: формування плодових тіл, лікарські гриби, чиста культура, рідкісні види, склероцієподібні структури. 
Lesia Ukrainka Eastern European National University Scientific Bulletin Series:

Biological Sciences, 2019,3 (387)

\section{Introduction}

More than 5000 species of Ascomycetes and Agaricales fungi are known nowadays. Only approximately 2,000 species are considered edible (Lakhanpal, Rana, 2005). However, 30 species of them are intensively grown and have been entered into the culture (Akata et al., 2012; Buchalo, 1988; Krupodorova et al., 2012).

Basidiomes and mycelium include various nutritional substances that are used in food, medicine, cosmetics, etc. (Ikekawa et al., 2004; Gao et al., 2005; Carvaja et al.1, 2012). Medical properties were described for many macromycetes (Dovgiy et al., 2013; Krupodorova et al., 2012; Solomko, Lomberg, 2005; Wasser, 2010). Recently, the biosynthesis of fungal biologically active substances with immunomodulation, radioprotective, antitumor, antiviral and other properties is an investigation by many scientists (Engler, 1998; Takaku et al., 2001; Zjawiony 2004; Mariga et al., Vaz et al., 2010; Jeena et al., 2014). The medicinal mushrooms products derived from basidiomes have been commercially grown or pick up from the wild. Only $15 \%$ of all products of medical mushrooms are made from extracts of mycelium (Lindequist et al., 2005). However, in our opinion, almost all mushrooms may be medical, but we don't know it precisely yet.

Now scientists are studying intensely fungi but their special interest is an investigation of mycorrhizal fungi (Sanmee et al., 2010; Akata et al., 2012; Endo et al., 2014). One of the reasons a small number of species that can be grown in industrial culture are unclear conditions for the basidioma formation. Verification of conditions mushrooms cultivation will significantly expand the range of species that could be a potential object for biotechnology. So, the study of the mushrooms basidiomes formation in culture is important in many aspects. The purpose of our work was the initiation of basidioma formation of rare and medical mushrooms in culture.

\section{Materials and Methods}

The 29 species of macromycetes from the pure Cultures Collection of Fungi (FCKU) of Educational and Scientific Centre Institute of «Biology and Medicine» Taras Shevchenko National University of Kyiv have used in our experiment (tab.1). Collection FCKU was registered in the World Federation of Cultures collections and includes 51 species: 19 species of them are edible, 5 - inedible, 1 - poisonous, 4 - included in the Red Data Book of Ukraine (2009) and 4 are rare species that need additional protection.
Species names were agreed according to the current names in the International Database of Mushrooms. The cultures are stored in test tubes with a potato-glucose agar medium (PGA) in an equipped room, 4-8 ${ }^{\circ} \mathrm{C}$ temperature. We used different nutrient media, substrates and substrates combinations for the basidiomes formation.

The nutrient media are potato-glucose agar medium (PGA) and liquid potato-glucose nutrient medium (PG).

The substrates are husks of pumpkin, husk of sunflower seeds, boiled wheat grains, pine sawdust, ground coffee beans.

The substrate combinations are mixtures of ground coffee beans and husk of sunflower seeds, a mix of pine sawdust with husk of sunflower seeds, a mix of a husk of sunflower seeds with sawdust of hardwoods.

$50 \mathrm{~g}$ substrates have put into $250 \mathrm{ml}$ Erlenmeyer flask or flat-bottomed round flask. Each flask with substrates was filled with $50 \mathrm{ml}$ of water. The moisture content of the solid media was adjusted to $65 \%(\mathrm{w} / \mathrm{w})$ on a wet basis. $50 \mathrm{~g}$ of the liquid potato-glucose nutrient medium have poured into $250 \mathrm{ml}$ flat-bottomed round flask. All nutrient media, substrates and substrate combinations were prepared according to generally accepted methods and were autoclaved at $120^{\circ} \mathrm{C}$ for $30 \mathrm{~min}$. (Buchalo, 1988; Ohta and Fujiwara, 2003).

Mycelia agar-plugs (3-5 $\mathrm{mm}$ diam.) cut from the periphery of the growing colony and were put to flasks with nutrient media, substrates and substrate combinations. After then, inoculated flasks were incubated in darkness at an optimal temperature for mycelium growing of these species. When the surface of the medium was covered by mycelium, incubation conditions were changed to daylight with temperature to $16-18^{\circ} \mathrm{C}$ for fruit body formation. Maximum light intensity was 1600-2000 lux daily.

The Thiamine (Vitamin B1) $(0,25 \mathrm{mg}$ added to each flask) or selenium (Se) $(0,125 \mathrm{~g}$ in each flask) have put in some substrates for initiation basidiomes formation. The beginning of basidiomes formation was determined of primordium appearance.

Same basidiomes of studying mushrooms have formed spores that were checking by microscope. We used statistical analysis to test our data. The entire process was repeated with five replicates.

\section{Results}

The investigated species of fungi, their location source and the success of basidiomes formation in vitro are shown in table 1. 
Successful of Basidiomes Formations of Studied Macromycetes in Vitro

\begin{tabular}{|c|c|c|c|c|c|}
\hline Species & Origin & $\begin{array}{c}\text { Date } \\
\text { of Collection }\end{array}$ & Edibility & Rarity & $\begin{array}{c}\text { Basidiomes } \\
\text { in Pure } \\
\text { Culture } \\
\end{array}$ \\
\hline 1 & 2 & 3 & 4 & 5 & 6 \\
\hline $\begin{array}{c}\text { Ascocoryne } \\
\text { sarcoides }\end{array}$ & Kiev region & September, 2010 & NK & & $\mathrm{F}$ \\
\hline $\begin{array}{l}\text { Armillaria } \\
\text { mellea }\end{array}$ & $\begin{array}{c}\text { Kherson region, } \\
\text { Chornomorsky State } \\
\text { Biosphere Reserve }\end{array}$ & October, 2006 & $\mathrm{E}$ & & $\mathrm{SS}, \mathrm{R}$ \\
\hline $\begin{array}{c}\text { Ceriporia } \\
\text { viridans } \\
\end{array}$ & $\begin{array}{c}\text { Kaniv, Cherkasy region, } \\
\text { Kaniv Nature Reserve }\end{array}$ & June, 2012 & $\mathrm{NE}$ & & $\mathrm{F}$ \\
\hline $\begin{array}{c}\text { Chondrostereum } \\
\text { purpureum }\end{array}$ & Kiev region & September, 2008 & $\mathrm{NE}$ & & SS \\
\hline $\begin{array}{l}\text { Coprinus } \\
\text { micaceus } \\
\end{array}$ & $\begin{array}{c}\text { Volnovakha district, } \\
\text { Donetsk region }\end{array}$ & June, 2005 & $\mathrm{E}$ & & $\mathrm{F}$ \\
\hline $\begin{array}{l}\text { Coprinellus } \\
\text { domesticus }\end{array}$ & $\begin{array}{c}\text { Kaniv, Cherkasy region, } \\
\text { Kaniv Nature Reserve }\end{array}$ & June, 20011 & $\mathrm{NE}$ & & $\mathrm{F}$ \\
\hline Grifola frondosa & $\begin{array}{c}\text { Sloviansky district, } \\
\text { Donetsk region }\end{array}$ & October, 2003 & $\mathrm{E}$ & $\mathrm{RB}$ & $\mathrm{F}$ \\
\hline $\begin{array}{l}\text { Hericium } \\
\text { cirrhatum } \\
\end{array}$ & $\begin{array}{c}\text { Kaniv, Cherkasy region, } \\
\text { Kaniv Nature Reserve }\end{array}$ & June, 2008 & $\mathrm{E}$ & $\mathrm{R}$ & $\mathrm{F}$ \\
\hline $\begin{array}{l}\text { Hypholoma } \\
\text { fasciculare }\end{array}$ & Kiev region & October, 2011 & $\mathrm{P}$ & & $\mathrm{F}$ \\
\hline $\begin{array}{l}\text { Hypholoma } \\
\text { sublateritium }\end{array}$ & Kiev region & October, 2011 & $\mathrm{NE}$ & & $\mathrm{F}$ \\
\hline $\begin{array}{c}\text { Lentinus } \\
\text { cyathiformis }\end{array}$ & $\begin{array}{c}\text { Kaniv, Cherkasy region, } \\
\text { Kaniv Nature Reserve }\end{array}$ & June, 2008 & $\mathrm{NE}$ & & SS \\
\hline Lentinus tigrinus & Cherkasy region & June, 2007 & $\mathrm{E}$ & & $\mathrm{F}$ \\
\hline $\begin{array}{l}\text { Leucoagaricus } \\
\text { barsii }\end{array}$ & $\begin{array}{l}\text { Trakhtemyriv village, } \\
\text { Kaniv distric, Cherkasy } \\
\text { region }\end{array}$ & November, 2008 & $\mathrm{E}$ & $\mathrm{RB}$ & $\mathrm{F}$ \\
\hline $\begin{array}{l}\text { Marasmius } \\
\text { scorodonius }\end{array}$ & Kiev region & September, 2009 & $\mathrm{E}$ & & $\mathrm{F}$ \\
\hline $\begin{array}{l}\text { Meripilus } \\
\text { giganteus }\end{array}$ & $\begin{array}{c}\text { Volnovakha distric, } \\
\text { Donetsk region }\end{array}$ & September, 2005 & $\mathrm{NE}$ & & $\mathrm{F}$ \\
\hline $\begin{array}{c}\text { Morchella } \\
\text { crassipes }\end{array}$ & $\begin{array}{c}\text { Kaniv, Cherkasy region, } \\
\text { Kaniv Nature Reserve }\end{array}$ & May, 2008 & $\mathrm{E}$ & $\mathrm{RB}$ & $\mathrm{SS}$ \\
\hline Morchella elata & Lutsk & May, 2009 & $E$ & & $\mathrm{SS}$ \\
\hline $\begin{array}{c}\text { Morchella } \\
\text { esculenta }\end{array}$ & Kiev region, & April, 2012 & $\mathrm{E}$ & & SS \\
\hline $\begin{array}{l}\text { Morchella } \\
\text { steppicola }\end{array}$ & $\begin{array}{c}\text { Volnovakha district, } \\
\text { Donetsk region }\end{array}$ & May, 2004 & $\mathrm{E}$ & $\mathrm{RB}$ & SS \\
\hline Mutinus caninus & $\begin{array}{c}\text { Kaniv, Cherkasy region, } \\
\text { Kaniv Nature Reserve }\end{array}$ & June, 2007 & $\mathrm{NE}$ & $\mathrm{RB}$ & SS \\
\hline $\begin{array}{c}\text { Oudemansiella } \\
\text { longipes }\end{array}$ & $\begin{array}{c}\text { Kyiv, Holosiyvsky } \\
\text { National Nature Park }\end{array}$ & September, 2013 & $\mathrm{NE}$ & & $\mathrm{F}$ \\
\hline $\begin{array}{l}\text { Oudemansiella } \\
\text { radicata }\end{array}$ & $\begin{array}{c}\text { Kaniv, Cherkasy region, } \\
\text { Kaniv Nature Reserve }\end{array}$ & June, 2007 & $\mathrm{E}$ & & $\mathrm{SS}$ \\
\hline $\begin{array}{c}\text { Phallus } \\
\text { impudicus }\end{array}$ & $\begin{array}{c}\text { Kaniv, Cherkasy region, } \\
\text { Kaniv Nature Reserve }\end{array}$ & June, 20011 & $\mathrm{E}$ & & $\mathrm{N}$ \\
\hline $\begin{array}{l}\text { Pleurotus } \\
\text { calyptratus }\end{array}$ & Kiev region & October, 2012 & $\mathrm{E}$ & & $\mathrm{SS}$ \\
\hline $\begin{array}{l}\text { Polyporus } \\
\text { squamosus }\end{array}$ & $\begin{array}{l}\text { Sosnytsia district, } \\
\text { Chernihiv region }\end{array}$ & October, 2010 & $\mathrm{E}$ & & $\mathrm{F}$ \\
\hline
\end{tabular}




\begin{tabular}{|c|c|c|c|c|c|}
\hline $\mathbf{1}$ & $\mathbf{2}$ & $\mathbf{3}$ & $\mathbf{4}$ & $\mathbf{5}$ & $\mathbf{6}$ \\
\hline $\begin{array}{c}\text { Pycnoporus } \\
\text { cinnabarinus }\end{array}$ & Kiev region & September, 2011 & NE & F \\
\hline $\begin{array}{c}\text { Sarcodontia } \\
\text { crocea }\end{array}$ & $\begin{array}{c}\text { Sosnytsia district, } \\
\text { Chernihiv region }\end{array}$ & October, 2010 & NE & R & F \\
\hline Sparassis crispa & Kiev region & September, 2013 & E & RB & F \\
\hline $\begin{array}{c}\text { Sparassis } \\
\text { laminosa }\end{array}$ & $\begin{array}{c}\text { Artemivsk district, } \\
\text { Donetsk region }\end{array}$ & September, 1996 & E & R & F \\
\hline
\end{tabular}

Note. Edibility: $E$ - edible species, $N$-inedible species, $O$ - poisonous species; Rarity: $R$ - rare species, $R B$ - species listed in the Red Data Book of Ukraine; Successful fruit formation: SS - species that formed primordia or sklerotium-shaped structures, F- species, which formed basidiomes in a pure culture, $N$ - species that did not form basidiomes.

Primarily, all of investigated species have basidiomes formation on the three type of substrates such as husk of sunflower seeds, PGA and PG. The time (days) for overgrowth of the substrate by mycelium, appearance of primordia and basidiomes of all species are shown in table 2 .

Fouling Substrate and Basidiomes Formation of Studied Species (Days)

Table 2

\begin{tabular}{|c|c|c|c|c|c|c|c|c|c|}
\hline \multirow[b]{3}{*}{ Species } & \multicolumn{9}{|c|}{ Type of Substrates } \\
\hline & \multicolumn{3}{|c|}{ PGA } & \multicolumn{3}{|c|}{ PG } & \multicolumn{3}{|c|}{ Husk of Sunflower Seeds } \\
\hline & 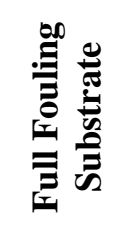 & 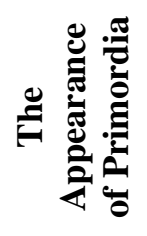 & 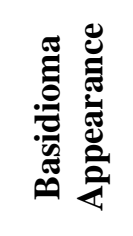 & 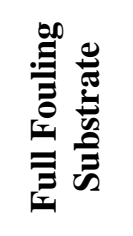 & 恶 & 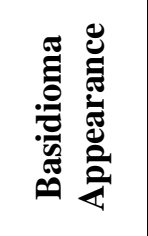 & 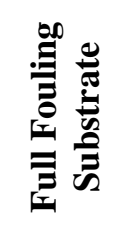 & 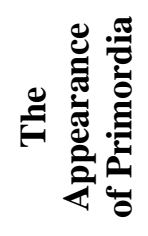 & 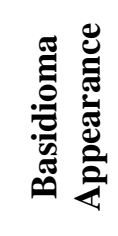 \\
\hline 1 & 2 & 3 & 4 & 5 & 6 & 7 & 8 & 9 & 10 \\
\hline $\begin{array}{l}\text { Ascocoryne } \\
\text { sarcoides }\end{array}$ & $10 \pm 0,6$ & $14 \pm 0,3$ & $16 \pm 0,3$ & $13 \pm 0,9$ & - & - & $14 \pm 0,4$ & $20 \pm 0,9$ & $34 \pm 1,2$ \\
\hline Armillaria mellea & $28 \pm 2,5$ & $36 \pm 1,5$ & - & $32 \pm 3,5$ & - & - & $43 \pm 0,2$ & $56 \pm 1,3$ & - \\
\hline $\begin{array}{c}\text { Morchella } \\
\text { crassipes }\end{array}$ & $5 \pm 0,7$ & $14 \pm 1,3$ & - & $6 \pm 0,8$ & - & - & $13 \pm 0,3$ & $2 \pm 0,4$ & - \\
\hline Morchella elata & $6 \pm 0,9$ & $16 \pm 1,3$ & - & $7 \pm 0,9$ & $20 \pm 0,4$ & - & $13 \pm 0,3$ & $21 \pm 0,4$ & - \\
\hline $\begin{array}{c}\text { Morchella } \\
\text { esculenta }\end{array}$ & $7 \pm 0,4$ & $18 \pm 1,3$ & - & $9 \pm 0,4$ & - & - & $13 \pm 0,3$ & $21 \pm 0,4$ & - \\
\hline $\begin{array}{l}\text { Morchella } \\
\text { steppicola }\end{array}$ & $8 \pm 0,3$ & $21 \pm 2,3$ & - & $9 \pm 0,3$ & - & - & $13 \pm 0,3$ & $21 \pm 0,4$ & - \\
\hline $\begin{array}{c}\text { Chondrostereum } \\
\text { purpureum }\end{array}$ & $8 \pm 0,8$ & - & - & $8 \pm 1,5$ & - & - & $10 \pm 0,3$ & $32 \pm 0,2$ & - \\
\hline $\begin{array}{l}\text { Coprinus } \\
\text { micaceus } \\
\end{array}$ & $14 \pm 0,4$ & $20 \pm 0,3$ & $21 \pm 0,8$ & $16 \pm 0,7$ & - & - & $19 \pm 0,9$ & $25 \pm 0,6$ & $26 \pm 0,2$ \\
\hline $\begin{array}{c}\text { Coprinellus } \\
\text { domesticus }\end{array}$ & $13 \pm 0,5$ & $19 \pm 0,3$ & $20 \pm 0,4$ & $14 \pm 0,5$ & - & - & $19 \pm 0,6$ & $24 \pm 0,2$ & $25 \pm 0,2$ \\
\hline Grifola frondosa & $15 \pm 0,8$ & - & - & $80 \pm 0,3$ & $108 \pm 0,2$ & $118 \pm 0,9$ & $25 \pm 0,7$ & $76 \pm 0,2$ & $79 \pm 0,9$ \\
\hline Ceriporia viridans & $15 \pm 1,0$ & $25 \pm 0,7$ & $32 \pm 1,2$ & $22 \pm 1,1$ & - & - & $24 \pm 1,6$ & $33 \pm 2,2$ & $44 \pm 2,8$ \\
\hline $\begin{array}{l}\text { Hericium } \\
\text { cirrhatum } \\
\end{array}$ & $14 \pm 0,5$ & $18 \pm 0,8$ & $21 \pm 0,7$ & $35 \pm 0,6$ & $41 \pm 0,7$ & $45 \pm 0,3$ & $20 \pm 0,5$ & $25 \pm 0,9$ & $30 \pm 0,5$ \\
\hline $\begin{array}{l}\text { Hypholoma } \\
\text { fasciculare }\end{array}$ & $18 \pm 0,9$ & - & - & $30 \pm 0,2$ & - & - & $33 \pm 0,1$ & $39 \pm 0,2$ & $45 \pm 0,8$ \\
\hline $\begin{array}{c}\text { Hypholoma } \\
\text { sublateritium }\end{array}$ & $33 \pm 1,7$ & $22 \pm 1,9$ & $47 \pm 2,9$ & $32 \pm 1,3$ & - & - & $42 \pm 2,2$ & $48 \pm 2,4$ & $54 \pm 1,8$ \\
\hline
\end{tabular}


Науковий вісник Східноєвропейського національного університету імені Лесі Українки. Серія: Біологічні науки, 2019, 3 (387)

Continuation of Table 2

\begin{tabular}{|c|c|c|c|c|c|c|c|c|c|}
\hline 1 & 2 & 3 & 4 & 5 & 6 & 7 & 8 & 9 & 10 \\
\hline Lentinus tigrinus & $11 \pm 0,3$ & $20 \pm 0,6$ & $29 \pm 1,8$ & $43 \pm 0,3$ & - & - & $14 \pm 0,3$ & $35 \pm 0,9$ & $39 \pm 0,3$ \\
\hline $\begin{array}{c}\text { Lentinus } \\
\text { cyathiformis }\end{array}$ & $14 \pm 0,5$ & - & - & $18 \pm 1,8$ & - & - & $22 \pm 0,2$ & $137 \pm 0,4$ & - \\
\hline $\begin{array}{c}\text { Leucoagaricus } \\
\text { barsii * }\end{array}$ & $19 \pm 1,3$ & - & - & $16 \pm 2,6$ & - & - & $32 \pm 2,3$ & $91 \pm 4,9$ & $96 \pm 5,3$ \\
\hline $\begin{array}{c}\text { Marasmius } \\
\text { scorodonius } *\end{array}$ & $15 \pm 0,7$ & - & - & $49 \pm 0,3$ & - & - & $31 \pm 0,3$ & $37 \pm 0,9$ & $41 \pm 0,3$ \\
\hline $\begin{array}{l}\text { Meripilus } \\
\text { giganteus }\end{array}$ & $10 \pm 0,7$ & $14 \pm 1,2$ & $20 \pm 0,9$ & $13 \pm 0,3$ & - & - & $10 \pm 0,3$ & $16 \pm 0,8$ & $18 \pm 0,5$ \\
\hline Mutinus caninus & $10 \pm 0,4$ & $21 \pm 0,6$ & - & $12 \pm 0,3$ & $24 \pm 0,9$ & - & $16 \pm 1,2$ & $28 \pm 1,7$ & - \\
\hline $\begin{array}{c}\text { Oudemansiella } \\
\text { longipes }\end{array}$ & $14 \pm 0,4$ & - & - & $21 \pm 2,8$ & - & - & $18 \pm 1,4$ & $31 \pm 2,5$ & $37 \pm 3,1$ \\
\hline $\begin{array}{c}\text { Oudemansiella } \\
\text { radicata }\end{array}$ & $12 \pm 0,6$ & $32 \pm 1,7$ & - & $22 \pm 3,4$ & - & - & $16 \pm 0,4$ & $23 \pm 0,2$ & - \\
\hline Phallus impudicus & $32 \pm 3,4$ & - & - & $23 \pm 2,3$ & - & - & $25 \pm 2,4$ & - & - \\
\hline $\begin{array}{c}\text { Pleurotes } \\
\text { calyptratus }\end{array}$ & $10 \pm 0,7$ & - & - & $18 \pm 1,3$ & - & - & $11 \pm 1,3$ & $132 \pm 0,3$ & - \\
\hline $\begin{array}{l}\text { Polyporus } \\
\text { squamosus }\end{array}$ & $10 \pm 0,4$ & $20 \pm 1,3$ & - & $16 \pm 0,8$ & - & - & $17 \pm 0,1$ & $35 \pm 0,3$ & $43 \pm 0,1$ \\
\hline $\begin{array}{l}\text { Pycnoporus } \\
\text { cinnabarinus }\end{array}$ & $9 \pm 0,8$ & $20 \pm 1,5$ & $25 \pm 2,1$ & $20 \pm 0,7$ & $32 \pm 0,3$ & $53 \pm 1,5$ & $14 \pm 0,5$ & $50 \pm 0,7$ & $63 \pm 0,1$ \\
\hline $\begin{array}{c}\text { Sarcodontia } \\
\text { crocea }\end{array}$ & $17 \pm 0,5$ & $25 \pm 0,7$ & $30 \pm 0,7$ & $20 \pm 0,3$ & - & - & $25 \pm 0,8$ & $36 \pm 0,7$ & $41 \pm 0,9$ \\
\hline Sparassis crispa & $16 \pm 0,2$ & $20 \pm 0,7$ & $23 \pm 0,7$ & $22 \pm 0,4$ & $41 \pm 0,7$ & $46 \pm 0,7$ & $17 \pm 0,1$ & $35 \pm 0,3$ & $43 \pm 0,1$ \\
\hline $\begin{array}{c}\text { Sparassis } \\
\text { laminosa } \\
\end{array}$ & $16 \pm 0,2$ & - & - & $13 \pm 0,5$ & - & - & $23 \pm 0,2$ & $25 \pm 1,8$ & $33 \pm 0,4$ \\
\hline
\end{tabular}

Note. Column «The appearance of primordial» contains the results of appearance primordia or sclerotium-shaped structures; *-macromycetes generated sporocarps after coverage by soil and additional moisture into medium.

According to the table 2, M. gianteus had the fastest mycelium growing. Mycelium of this mushroom wholly covered the sunflower husk substrate and PG medium during 10 and 13 days of cultivations respectively. F. velutipes had the slowest mycelium growing. The sunflower husk substrate have had completely covered F. velutipes mycelium after 66 days cultivations and 45 days after PG medium cultivation accordingly.

Mycelium of almost investigated species, except Phallus impudicus L., was forming primordias and sclerotium-shaped structures on the tested substrates (fig.1).

Some species formed primordia even before full fouling of the substrate by the mycelium. M. giganteus have formed primordia on the sunflower husk substrate the fasted amount investigation mushrooms after 16 days of cultivations.

Ascocoryne sarcoides (Jacq.) J. W.Groves \& D. E. Wilson, Coprinus micaceus (Bull.: Fr.) Vilgalys, Hopple \& Jacq. Johnson, Coprinellus domesticus (Bolton) Vilgalys, Hopple \& Jacq.Johnson, Grifola frondosa (Dicks.) Gray,
Hericium cirrhatum (Pers.) Nikol., Hypholoma fasciculare (Huds.: Fr.) P. Kumm., Lentinus tigrinus (Bull.) Fr., Leucoagaricus barsii (Zeller) Vellinga, Marasmius scorodonius (Fr.) Fr., Meripilus giganteus Karst., Oudemansiella longipes (Quél.) M. M. Moser, Pycnoporus cinnabarinus (Jacq.) P. Karst., Sarcodontia crocea (Schwein.) Kotl., Sparassis crispa (Wulfen) Fr. and Sparassis laminosa Fries formed a well-developed basidiomes on the husk of sunflower seeds (fig. 2).

The early basidiomes of $\mathrm{M}$. giganteus looked like light-coloured corals, but later they acquired in a dark red colour. The most massive and intensive basidiomes formation had in $\mathrm{P}$. cinnabarinus (fig. 2 D) on the husk of sunflower seeds substrate. This fungus had basidiomes bright orange colour on the walls of a flask.

As we see from Figure 2 basidiomes of L. tigrinus has a stem of 3-4 cm length and cap of $2-6 \mathrm{~cm}$ diameter. The basidiomes of $S$. crocea looked like sharp spines of creamy colour with 1 to $2 \mathrm{~cm}$ in size and located in groups throughout the substrate surface (fig. 2 F). 

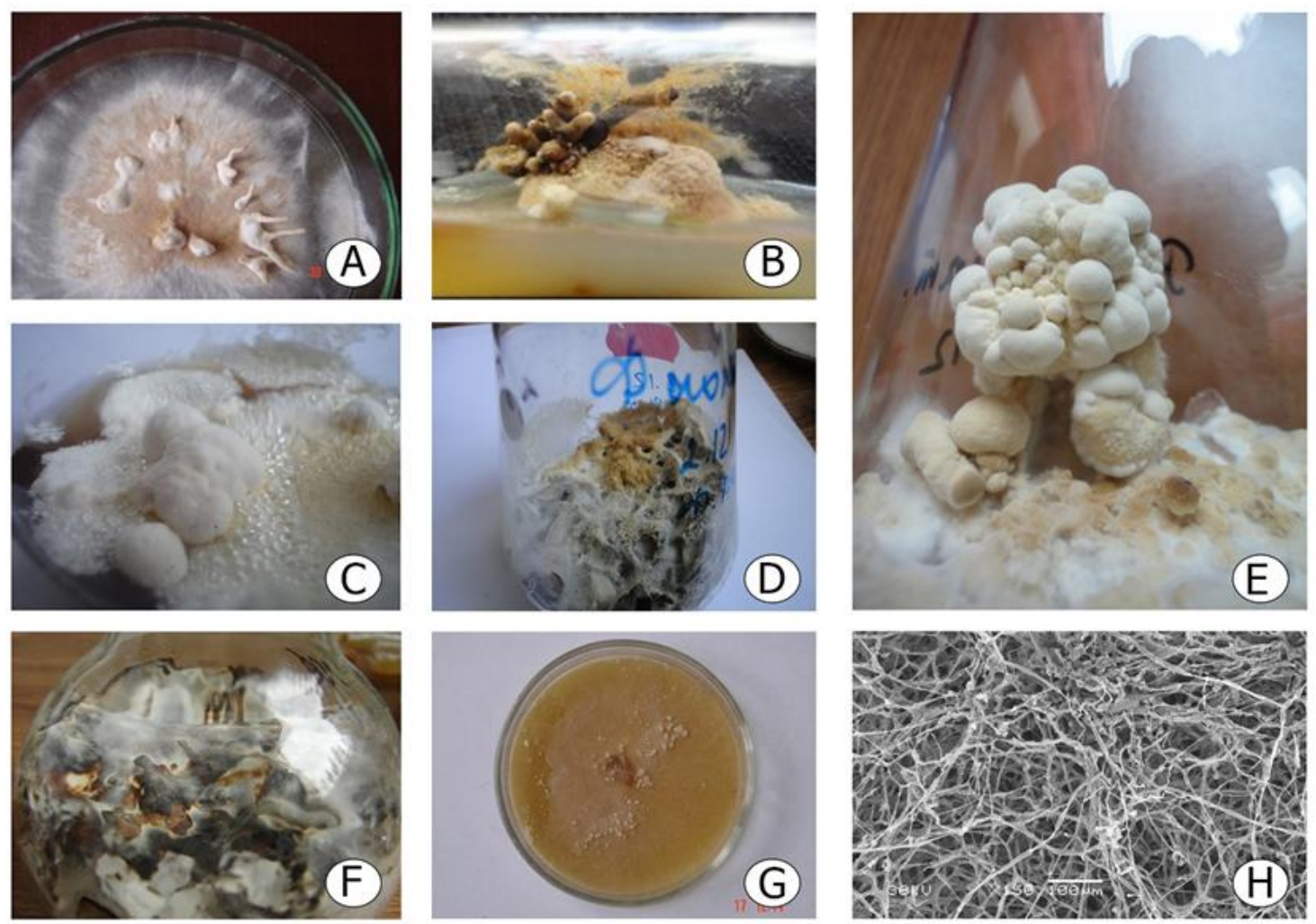

Fig. 1. Primordium and Sclerotium-Shaped Structure of Macromycetes in Culture. Primordia of Lentinus Tigrinus (A), Polyporus Squamosus (E), Hypholoma Sublateritium (B). Sclerotium-Shaped Structure of Mutinus Caninus (C), Chondrostereum Purpureum (D), Oudemansiella Radicata (F), Morchella Steppicola $(G)$ and Hyphae Sclerotium-Shaped Structure on a Scanning Electron Microscope $(H)$
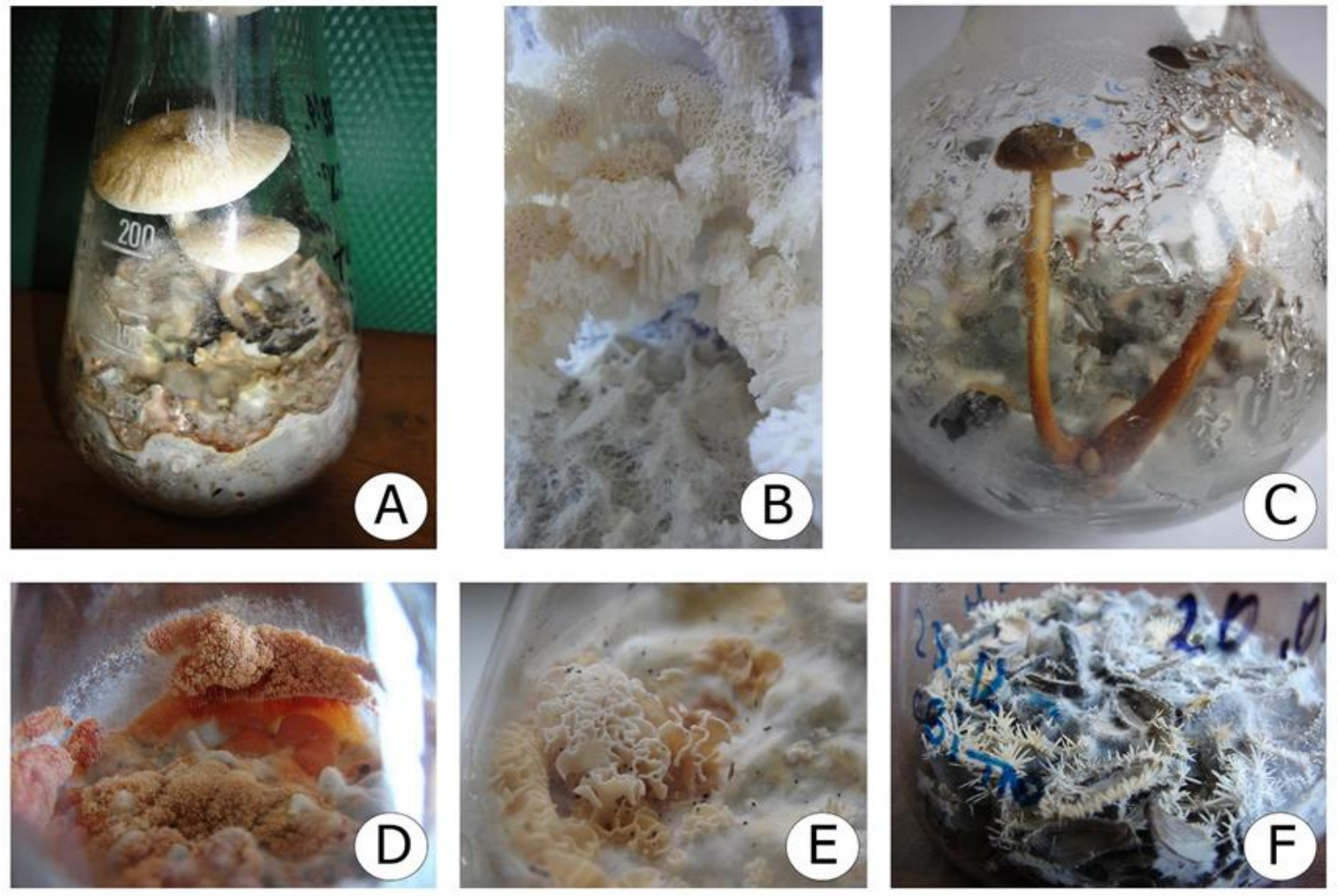

Fig. 2. Basidiomes Formation of Lentinus Tigrinus (A), Sparassis Laminosa (B), Oudemansiella Longipes (C), Pycnoporus Cinnabarinus (D), Grifola Frondosa (E), and Sarcodontia Crocea $(F)$ on Husk of Sunflower Seeds 

імені Лесі Украӥнки. Серія: Біологічні науки, 2019, 3 (387)

M. scorodonius formed brown basidiomes with a stem of $4-5 \mathrm{~cm}$ length and cap of $1 \mathrm{~cm}$ diameter (fig. 3 A). Also, this fungus had a garlic smell which inherent in these species. Some macromycetes generated sporocarps after additional coverage by soil and added moistures (fig. $3 \mathrm{~A}$ and $\mathrm{C}$ ). Several species developed basidiomes on liquid medium PG or PGA (fig. 3 B, D, E, F).

$H$. cirrhatum formed basidiomes on all type of tested media. The basidiomes look likes a white colour plate on a liquid nutrient medium and husk of sunflower seeds substrate. Also, this plate was twisted at the ends and formed small spines in the center of the plate (fig. $3 \mathrm{D}$ ). basidiomes on PGA had the form elongated strands with white color spines (fig. $3 \mathrm{~F}$ ).

The ten of twenty-nine studied species don't form basidiomes.

The basidiomes formation of rare macromycetes $S$. laminosa and $G$. frondosa was investigated in different substrates more details (table 3).

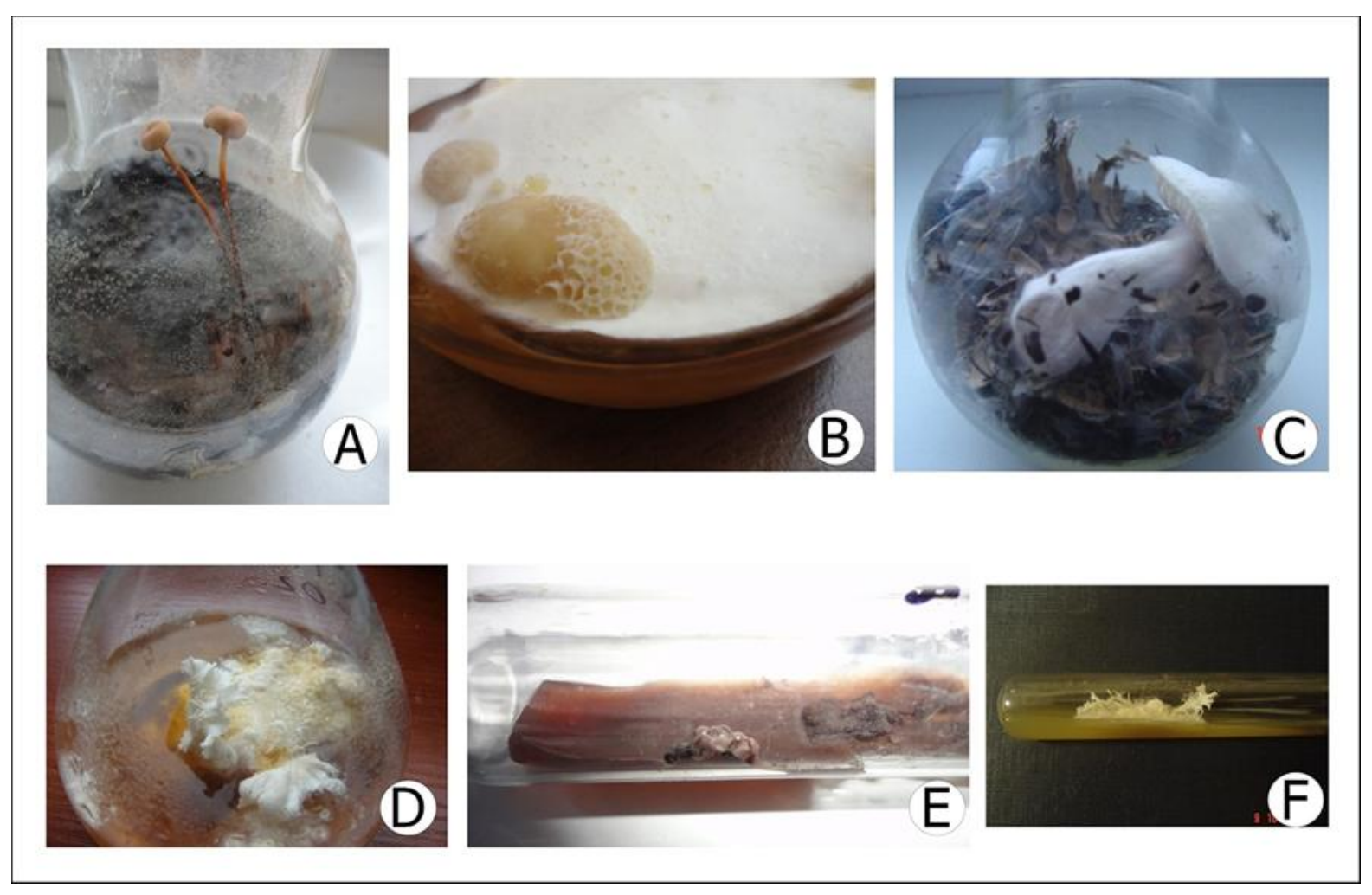

Fig. 3. Generation of Sporocarps After Additional Coverage By Soil and Additional Moisture by Marasmius Scorodonius (A) and Leucoagaricus Barsii (C). Basidiomes Formation by Grifola Frondosa (B), Hericium Cirrhatum (D) on Liquid Medium (PG); Ascocoryne Sarcoides (E), Hericium Cirrhatum $(F)$ on PGA

Table 3

Fouling Substrate and Basidiomes Formation by S. Laminosa and G. Frondosa (Days)

\begin{tabular}{|c|c|c|c|c|c|c|}
\hline \multirow{2}{*}{$\begin{array}{c}\text { Type } \\
\text { of } \\
\text { Substrates }\end{array}$} & $\begin{array}{c}\text { Full } \\
\text { Fouling } \\
\text { Substrate }\end{array}$ & $\begin{array}{c}\text { The } \\
\text { Appearance } \\
\text { of Primordia }\end{array}$ & $\begin{array}{c}\text { Basidioma } \\
\text { Appearance }\end{array}$ & $\begin{array}{c}\text { Full Fouling } \\
\text { Substrate }\end{array}$ & $\begin{array}{c}\text { The } \\
\text { Appearance } \\
\text { of Primordia }\end{array}$ & $\begin{array}{c}\text { Basidioma } \\
\text { Appearance }\end{array}$ \\
\hline HPS & $7 \pm 0,6$ & $24 \pm 1,9$ & - & $14 \pm 0,5$ & $40 \pm 0,8$ & $43 \pm 1,4$ \\
\hline PS+HSS & $11 \pm 0,4$ & $80 \pm 2,1$ & $85 \pm 2,9$ & $14 \pm 0,3$ & $70 \pm 0,2$ & $74 \pm 1,2$ \\
\hline H+HSS & $9 \pm 0,3$ & - & - & $14 \pm 1,3$ & $49 \pm 0,4$ & $53 \pm 0,6$ \\
\hline CB & $4 \pm 0,7$ & - & - & $12 \pm 0,8$ & - & - \\
\hline CB+HSS & $27 \pm 0,4$ & - & - & $33 \pm 0,5$ & $55 \pm 0,5$ & $59 \pm 0,4$ \\
\hline WG & $24 \pm 0,8$ & - & - & $32 \pm 0,5$ & $28 \pm 0,3$ & - \\
\hline
\end{tabular}

Note. HPS - husk of pumpkin seeds; WG - wheat grain; $C B-$ milled coffee beans; CB+HSS - mix of milled coffee beans with husk of sunflower seeds; PS+HSS - mix of pine sawdust with husk of sunflower seeds; $H+$ HSS - mix sawdust of hardwood with husk of sunflower seeds. 
The coffee beans substrate was the fastest covered by mycelium of $S$. laminosa and $G$. frondosa through 4 and 12 days respectively. However, on this substrate, S. laminosa and $G$. frondosa did not form primordia and basidiomes. Although S. laminosa and G. frondosa slowly overgrown substrates, but basidiomes formed at the faster on husk of sunflower seeds.

The only suitable substrate for fruiting $S$. laminosa was a mix of pine sawdust and husk of sunflower seeds. However, the basisiomes formation takes a long time (80 days) on a mix of pine sawdust with husk of sunflower seeds.

The favorable substrates for basidiomes formation by $G$. frondosa were pumpkin seed husks and a mix of hardwood sawdust and husk of sunflower seeds. Its basidiomes were as small bushes that were initially white, later becoming brown at all types of substrate. The largest number of basidiomes of $G$. frondosa was formed on a mix of pine sawdust with husk of sunflower seeds, but they were very small. Large basidiomes are formed on a mix of milled coffee beans with husk of sunflower seeds.

The effect of Thiamine (Vitamin B1) on the basidiomes formation was tested on S. laminosa. Vitamin B1 accelerated fouling substrate mycelium (10 $\pm 1,5$ days) and the mass of basidiomes. The term of basidiomes formation was $9 \pm 1,3$ days.

The added Selenium in each of the flasks with husk of sunflower seeds did not affect on the basidiomes formation of Mutinus caninus (Huds.) Fr., but speeded up fouling substrate by mycelium (average $9 \pm 2,0$ days).

\section{Discussion}

As a result screening of 29 macromycetes species on the ability to produce basidiomes in pure culture, the capacity of 28 of them was installed, including possibility to form primordia or sclerotium-shaped structures. Basidiomes were obtained in three species, which are included in the Red Data Book of Ukraine (G. frondosa, L. barsii, S. crispa) and four rare species (Ceriporia viridans (Berk. \& Broome) Donk, H. cirrhatum, S. crocea, $S$. laminosa) in pure culture.

The good substrate for the studied species appeared husk of sunflower seeds. Most fungi formed basidiomes on it. If mushrooms are not formed basidiomes on this substrate, they fastest it overgrown or developed primordia or sclerotiumshaped structures.

There is the positive effect of Vitamin B1 on the indicators of the basidiomes formation for example S. laminosa. The species that formed primordia or sclerotium-shaped structures (Armillaria mellea (Vahl) P. Kumm., Chondrostereum purpureum (Pers.) Pouzar, Lentinus cyathiformis (Schaeff.) Bres., Morchella crassipes (Vent.) Pers., Morchella elata Fr., Morchella esculenta Fr., Morchella steppicola Zerova, Mutinus caninus, Pleurotus calyptratus (Lindblad ex Fr.) Sacc., Polyporus squamosus (Huds.) Fr. and Oudemansiella radicata (Relhan) Singer) are promising for further research the initiation basidiomes formation in a pure culture.

\section{Reference}

1. Akata, I.; Kalyoncu, F.; Solak, M. H.; Kalmis, E. Growth of mycelium of three ectomycorrhizal macrofungi, Infundibulicybe geotropa, Tricholoma anatolicum and Lactarius deliciosus in culture media containing various carbon sources. African Journal of Microbiology Research; 2012, 6(12), 3042-3046.

2. Buchalo, A. S. Higher Edible Basidiomycetes in Pure Culture; Naukova Dumka: Kiev; 1988.

3. Carvajal, A. E. S. S.; Koehnlein, E. A.; Soares, A. A.; Eler, G. J.; Nakashima, A. T. A.; Bracht, A.; Peralta, R. M. Bioactives of fruiting bodies and submerged culture mycelia of Agaricus brasiliensis (A. blazei) and their antioxidant properties. LWT - Food Science and Technology; 2012, 46, 493-499.

4. Diduh, Ya. P. Red Book of Ukraine. Flora; Hlobalkonsaltynh: Kyiv, 2009.

5. Dovgiy, R. S.; Pozur, V. V.; Svyatetska, V. N.; Suhomlyn, M. N.; Livinska, O. P.; Makarenko, A. N. Immunobiological action of extracts and medicines, obtained from mushrooms Ganoderma lucidum, Cordyceps sinensis and Leucoagaricus macrorhizus. Bulletin of the problems of biology and medicine (In Ukraine); 2013, 2(2), 44-49.

6. Endo, N.; Kawamura, F.; Kitahara, R.; Sakuma, D.; Fukuda, M.; Yamada, A. Synthesis of Japanese Boletus edulis ectomycorrhizae with Japanese red pine. Mycoscience; 2014, 55(5), 405-416.

7. Engler, M.; Anke, T; Sterner, O. Production of antibiotics by Collybia nivalis, Omphalotus olearis, a Favolaschia and a Pterula species on natural substrates. Z Naturforsch; 1988, 53(5-6), 318-24.

8. Gao, Y.; Chan, E.; Zhou, S. Immunomodulating activities of Ganoderma, a mushroom with medicinal properties. Food Rev. Int.; 2004, 2(20), 123-161.

9. Ikekawa, T.; Ikekawa, A.; Shimada, F. Physiologically active substance EEM-S originating in mushrooms, process for producing the same and drugs. 2004, US 6783771 B2, Aug. 31.

10. Jeena, G. S.; Punetha, H.; Prakash, O.; Chandra, M.; Kushwaha, K. P. S. Study on in vitro antioxidant potential of some cultivated Pleurotus species (oyster mushroom). Indian Journal of Natural Products and Resources; 2014, 5(1), 56-61.

11. Krupodorova, T. A.; Barshteyn, V. Yu.; Bisko, N. A.; Ivanova, T. S. Some nutritional medicinal fungi cultivated on amaranthus seed meal-based Сухомлин М., Діденко В., Цвид Н., Петричук Ю. 
medium. International Journal of Medicinal Mushroom; 2012, 14(3), 295-293.

12. Lakhanpal, T. N.; Rana, M. Medicinal and nutraceutical genetic resources of mushrooms. Plant Genet. Resour. Charact. and Util; 2005, 2(3), 288-303.

13. Lindequist, U.; Niedermeyer, T. H. J.; Julich, W.-D. The pharmacological potential of mushrooms. Evidence-based Complementary and Alternative Medicine; 2005, 2, 285-299.

14. Mariga, A. M.; Yang, W.-j.; Mugambi, D. K.; Pei F.; Zhao, L.-y.; Shao, Y.-n. and Hu, Q. Antiproliferative and immunostimulatory activity of a protein from Pleurotus eryngii. J. Sci. Food Agric; 2014, 94(15), 3152-3162.

15. Ohta, A.; Fujiwara, N. Fruit-body production of an ectomycorrhizal fungus in genus Boletus in pure culture. Mycoscience; 2003, 44(4), 295-300.

16. Sanmee, R.; Lumyong, P.; Dell, B.; Lumyong, S. In vitro cultivation and fruit body formation of the black bolete, Phlebopus portentosus, a popular edible ectomycorrhizal fungus in Thailand. Mycoscience; 2010, 51(1), 15-22.

17. Solomko, E. F.; Lomberg, M. L. The Selection of Alternative Substrates for Medicinal. International Journal of Medicinal Mushrooms. 2005, 7, 466.
18. Takaku, T.; Kimura, Y.; Okuda, H. Isolation of an antitumor compound from Agaricus blazei Murill and its mechanism of action. The Journal of Nutrition; 2001, 131(5), 1409-1413.

19. Vaz, J.A.; Heleno, S.A.; Martins, A.; Almeida, G.M.; Vasconcelos, M.H.; Ferreira, I.C.F.R. Wild mushrooms Clitocybe alexandri and Lepista inversa: in vitro antioxidant activity and growth inhibition of human tumour cell lines. Food and Chemical Toxicology; 2010, 48, 2881-2884.

20. Walter S. Non-wood forest products in Africa: a regional and national overview. Les produits forestiers non ligneux en Afrique: un aperçu régional et national. FAO Forestry Department, Rome, August, 2001, Working Paper/Document de Travail nr. FOPW/01/1.

21. Wasser, S. P. Medicinal Mushroom Science: History, Current Status, Future Trends, and Unsolved Problems. International Journal of Medicinal Mushrooms; 2010, 12(1), 1-16.

22. Zjawiony, J. Biologically active compounds from Aphyllophorales (Polypore) fungi. J Nat Prod; 2004, 67, 300-10. 